\title{
The Mediating Role of Emotion Dysregulation and Problematic Internet Use in the Relationship Between Negative Affect and Excessive Daytime Sleepiness: A Structural Equation Model
}

\author{
Anna Guerrini Usubini ${ }^{1,2}$, Grazia Terrone ${ }^{3}$, Giorgia Varallo $\mathbb{D}^{1,2}$, Roberto Cattivelli ${ }^{4}$, \\ Giuseppe Plazzi $^{5,6}$, Gianluca Castelnuovo ${ }^{1,2}$, Adriano Schimmenti $\mathbb{D}^{7}$, Alessandro Musetti $\mathbb{1}^{8}$, \\ Christian Franceschini (iD) ${ }^{9}$ \\ 'Istituto Auxologico Italiano IRCCS, Psychology Research Laboratory, Milan, Italy; ${ }^{2}$ Department of Psychology, Catholic University of Milan, Milan, \\ Italy; ${ }^{3}$ Department of History, Cultural Heritage, Education and Society, University of Rome Tor Vergata, Rome, Italy; ${ }^{4}$ Department of Psychology, \\ University of Bologna, Bologna, 40I27, Italy; ${ }^{5}$ Department of Biomedical, Metabolic and Neural Sciences, University of Modena and Reggio Emilia, \\ Modena, 4I I25, Italy; ${ }^{6}$ IRCCS Institute of Neurological Sciences of Bologna (ISNB), Bologna, 40139, Italy; ${ }^{7}$ Faculty of Human and Social Sciences, \\ UKE-Kore University of Enna, Cittadella Universitaria, Enna, Italy; ${ }^{8}$ Department of Humanities, Social Sciences and Cultural Industries, University of \\ Parma, Parma, Italy; ${ }^{9}$ Department of Medicine and Surgery, University of Parma, Parma, Italy
}

Correspondence: Alessandro Musetti, Department of Humanities, Social Sciences and Cultural Industries, University of Parma, Borgo Carissimi I0, Parma, 43I2I, Italy, Tel +39 052I 034820, Email alessandro.musetti@unipr.it

Purpose: The present study aimed to explore the mediating role of emotion dysregulation and problematic Internet use in the relationship between negative affect and excessive daytime sleepiness.

Methods: This cross-sectional study included 664 Italian adults aged between 18 and 70 years $(\mathrm{M}=32.13$; $\mathrm{SD}=11.71)$. Participants were asked to complete the Depression Anxiety Stress Scale, the Difficulties in Emotion Regulation Scale, the Compulsive Internet Use Scale-7, and the Epworth Sleepiness Scale to assess negative affect, emotion dysregulation, problematic internet use, and excessive daytime sleepiness, respectively.

Results: According to our results there are significant associations between negative affect, emotion dysregulation, problematic Internet use, and daytime sleepiness. Moreover, the structural equation model showed good fit indices. Emotion dysregulation and problematic Internet use partially mediated the relationship between negative mood and excessive daytime sleepiness.

Conclusion: By exploring the role of emotion dysregulation on the association between excessive daytime sleepiness and its possible predictors, our study might represent an important step toward the implementation of psychological intervention for reducing excessive daytime sleepiness. Emotion dysregulation appears to play a significant role in explaining the relationship between negative affect, problematic Internet use, and excessive daytime sleepiness. Thus, it should be considered a treatment target for reducing excessive daytime sleepiness.

Keywords: negative affect, psychological distress, excessive daytime sleepiness, emotion dysregulation, problematic Internet use

\section{Introduction}

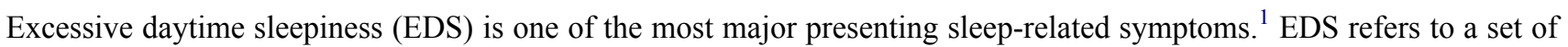
symptoms that include unplanned daytime sleep, as well as the inability to remain alert or awake in passive situations, ${ }^{2}$ resulting in an uncontrollable need to sleep and episodes of untimely sleep. EDS is the primary complaint of central disorders of hypersomnolence and is also a common symptom in both sleep apnea syndrome periodic limb movement disorder. $^{2,3}$ Furthermore, EDS significantly impair patients' quality of life, physical functioning, ${ }^{4,5}$ psychological wellbeing $^{5,6}$ and can disrupt social life $^{7}$ and work performance. ${ }^{8}$ 
Several factors contribute to EDS, including inadequate nocturnal sleep, the presence of underlying sleep disorders such as obstructive sleep apnea, narcolepsy, or circadian rhythm disturbances, ${ }^{9}$ as well as mood disorders (such as depression and anxiety). ${ }^{10}$

Indeed, an increasing number of studies have highlighted associations between depression, ${ }^{8,11-14}$ anxiety, ${ }^{15}$ stress, ${ }^{16}$ and EDS. Interestingly, a recent longitudinal study found that depression and anxiety were significant predictive factors for EDS in a sample of women in the general population. ${ }^{17}$

A potential underlying mechanism of the association between negative mood (ie, depression, anxiety, and stress) and $\mathrm{EDS}^{8,11-16,18}$ might be emotion regulation. According to Gratz and Roemer, ${ }^{19}$ emotion regulation is defined as the ability to be aware of and accept emotions, to behave in accordance with personal goals even in the presence of negative emotions, while controlling impulsive behaviors. Whereas adaptive emotion regulation strategies play a protective role and can promote resilience against stressful life events, ${ }^{20}$ on the contrary, dysfunctional emotion regulation is associated with several and significant forms of psychopathology. ${ }^{21}$ In this regard, given the association between negative mood and emotion dysregulation, ${ }^{22}$ it has been proposed that when functional emotion regulation strategies are lacking, experiencing negative affect (such as depression, anxiety, or stress) may lead to maladaptive behaviors, such as addictive behaviors. ${ }^{23,24}$

Nowadays, one of the most common addictive behaviors correlated to EDS is problematic Internet use.

Problematic Internet use (PIU) is defined as excessive time spent in online activities and is often accompanied by uncontrolled preoccupation, stress and computer-related urges ${ }^{25,26}$ that could lead to psychological and physical health issue. $^{26,27}$

Evidence highlighted several psychological factors associated with PIU, such as personality features, ${ }^{28}$ alexithymia ${ }^{29}$ and poorer psychological health. ${ }^{30}$ However, recently, emotional dysregulation has been suggested as a potential transdiagnostic factor underlying several psychopathological conditions ${ }^{31,32}$ including PIU. ${ }^{33,34}$ People with emotion regulation difficulties may engage in addictive behaviors in an attempt to minimize negative affect and emotional distress. ${ }^{34,35}$ In addition, PIU can be viewed as an "escape behavior" to cope with unpleasant feelings and emotions, ${ }^{36,37}$ aimed at alleviating negative moods. ${ }^{29,38}$ In line, one study found that individuals with PIU were more prone to suppressing and escaping their negative emotion engaging in PIU. ${ }^{39}$

It has been well documented that the excessive use of Internet could lead to many adverse health ${ }^{40,41}$ and psychological consequences, as well as sleeping problems and sleep deprivation. ${ }^{42}$ Specifically, one of the most serious sleep problems related to PIU is EDS. In a recent cross-sectional study, ${ }^{43}$ Demir et al found that PIU was an important factor that contributed to increasing the risk for EDS in a sample of University students. Generally, PIU is thought to lead to sleep deprivation. Indeed, it has been suggested that Internet use may cause a reduction in sleep duration ${ }^{44-46}$ and sleep onset difficulties, ${ }^{42,47}$ resulting in increased fatigue and sleepiness during the day.

The purpose of this study is to test the structural model, which was developed based on the literature to examine the relationships between negative affect, emotional dysregulation, PIU and EDS, as shown in Figure 1.

Although the relationship between PIU and EDS has received increasing attention in the literature, most studies involved adolescent and young adult samples, ${ }^{43,48,49}$ while evidence in adults is limited. ${ }^{50}$ Given these premises, the aim of the present study was to explore the relationship between negative affect and EDS, by assessing the mediating role of emotion dysregulation and PIU. We hypothesized that negative affect would be related to EDS and that this relationship would be partially and sequentially mediated by emotion dysregulation and PIU (Figure 1). Specifically, the hypotheses are the following:

H1: Negative affect levels would directly and positively affect emotional dysregulation levels.

H2: Emotional dysregulation levels would directly and positively affect PIU levels.

H3: PIU levels would directly and positively affect EDS levels.

H4: Negative affect levels would indirectly and positively affect EDS levels through emotional dysregulation and PIU. 


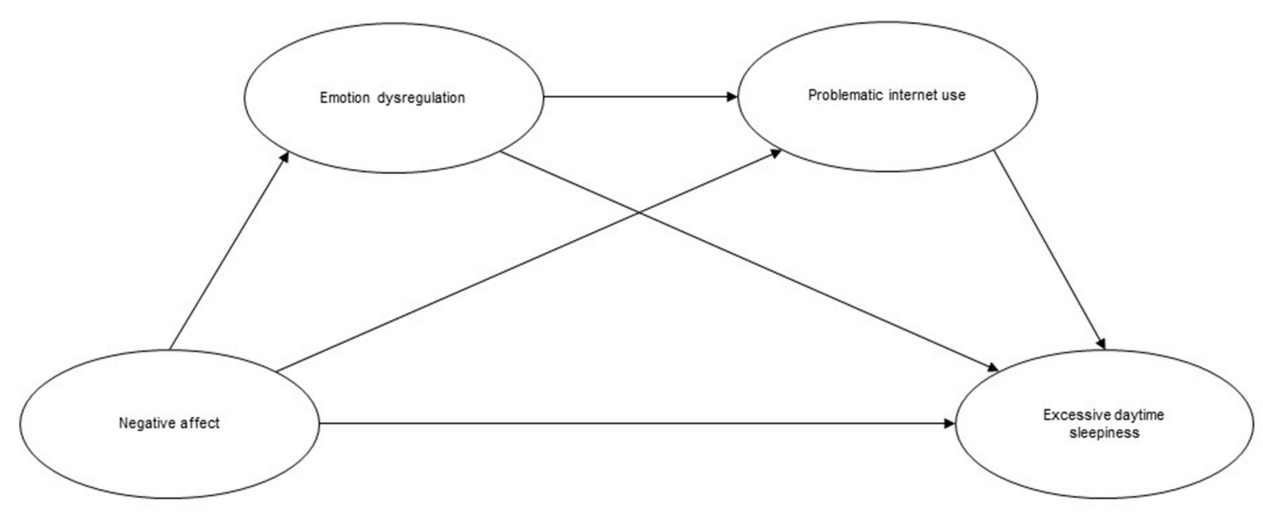

Figure I Hypothesized mediation model

\section{Materials and Methods \\ Participants and Procedures}

Data were collected from October to November 2020, during the COVID-19 pandemic. Participants were recruited from the general population via Internet announcements delivered on online platforms and social media. All participants were informed about the study and were asked to provide written informed consent. Participation in the study was entirely voluntary; participants were free to withdraw from the study at any time and did not receive any monetary compensation. All participants were asked to complete an online survey via an anonymous link. Uncompleted questionnaires were excluded from the data analysis. All procedures were carried out following the ethical standards of the Ethical Code of the Italian Association of Psychology (AIP), the European Code of Conduct for Research Integrity (ECCRI), the Helsinki Declaration of 1964, and its later advancements.

\section{Measures}

Demographical data were collected through a self-report form, while clinical factors were assessed using the following measures:

Negative affect. The Depression Anxiety Stress Scale (DASS-21) $)^{51}$ is a self-report questionnaire that assesses negative affect. The 21 items are rated on a 4-point Likert scale, ranging from 0 to 3. The DASS-21 is composed of three subscales: depression, anxiety, and stress. The total score ranges from 0 to 21, with higher scores indicating higher levels of negative affect. Scores $<10$ on the Depression subscale indicate normal levels of depression, while scores $>15$ indicate moderate-to-severe levels of depression. Scores $<8$ on the Anxiety subscale indicate normal levels of anxiety, while scores $>8$ indicate moderate-to-severe levels of depression. Scores $<15$ on the Stress subscale indicate normal levels of stress, while scores $>15$ indicate moderate-to-severe levels of stress. In our study, we used the Italian version ${ }^{52}$ which showed good psychometric properties (Cronbach's alpha values of subscales ranged from 0.83 to 0.91 ) in line with the original version. ${ }^{51}$ Cronbach's alpha in our sample was 0.92 .

Emotional dysregulation. The Difficulties in Emotion Regulation Scale (DERS) ${ }^{19}$ is a self-report questionnaire used to assess emotion dysregulation. It consists of 36 items, rated on a 5-point Likert scale, ranging from 1 to 5, with higher scores suggesting greater difficulties in emotion regulation. The DERS is divided into six subscales: non-acceptance of negative emotions, inability to engage in purposeful behavior when experiencing negative emotions, difficulty controlling impulsive behavior when experiencing negative emotions, limited access to emotion regulation strategies that are considered effective, lack of awareness of emotions, and lack of understanding of the nature of emotions. The Italian version $^{53}$ showed good psychometric properties. Cronbach's alpha of the total score was 0.92 . In our sample, Cronbach's alpha was 0.88 .

Excessive daytime sleepiness. The Epworth Sleepiness Scale (ESS) ${ }^{54}$ is a self-report questionnaire that assesses EDS. It is composed of 8 items ranging from 0 to 3 used. The total score ranges from 0 to 24 with higher scores 
indicating higher levels of EDS. Specifically, EES scores from 11 to 12 indicate Mild EDS; EES scores ranged from 13 to 14 indicate Moderate EDS; EES scores ranged from 16 to 24 indicate Severe EDS. We used the Italian version ${ }^{55}$ which showed good psychometric properties in line with the original version. ${ }^{54}$ The Cronbach's alpha in our sample was 0.71 .

Problematic Internet use. The Compulsive Internet Use Scale-7 (CIUS-7 $)^{56}$ is a self-report questionnaire that assesses PIU. It comprises 7 items ranging from 0 to 4 with higher scores indicating greater PIU severity. We used the Italian version validated by Lopez et al. ${ }^{57}$ In the current sample, Cronbach's alpha was 0.92 .

\section{Data Analytic Strategy}

Descriptive statistics, including frequencies and percentages for categorical variables and means and standard deviations for continuous variables, were computed. Normality assumptions were tested by skewness and kurtosis coefficients. Correlations between all the continuous study variables were evaluated using Pearson's correlations.

Subsequently, a structural equation model (SEM) was used to test the relationships between negative affect, emotional dysregulation, PIU, and EDS using Robust Maximum Likelihood estimator (RML). Latent variables for PIU and EDS were computed using items as indicators, whereas the latent variable for emotion dysregulation was calculated using the DERS subscales. Similarly, for negative affect, we used the DASS-21 subscales.

The model's goodness of fit was evaluated using the root-mean-square error of approximation (RMSEA), the comparative fit index (CFI), and the standardized root-mean-square residual (SRMR). Hu and Bentler's goodness-offit criteria ${ }^{58}$ were employed to indicate acceptable (CFI and Tucker-Lewis index [TLI] $>0.90$, SRMR $<0.10$, RMSEA $<$ $0.08)$ and excellent fit (CFI and TLI $>0.95$, SRMR $<0.08$, RMSEA $<0.06$ ).

Data were analyzed using IBM Statistical Package for the Social Sciences SPSS version 24 (Armonk, NY: IBM Corp) and Mplus software (version 8).

\section{Results}

\section{Descriptive Characteristics of the Sample and Correlations Between All the Variables of the Study}

This cross-sectional study included 664 Italian adults. The sample included 197 males (29.7\%) and 467 females (70.3\%). The mean age of the total sample was $32.13 \pm 11.71$ years in a range between 18 and 70 years. Specifically, among male participants the mean age was $32.84 \pm 10.7$ years in a range from a minimum of 18 to a maximum of 61 years. Among female participants the mean age was $31.82 \pm 12.11$ years in an age range from a minimum of 18 to a maximum of 70 years. Among participants, 596 (70.64\%) subjects reported no EDS, 68 (10.3\%) showed moderate-to-severe levels of EDS. As for negative affect, 524 participants (78.8\%) showed normal levels of depression and 140 (21.2\%) reported moderate-to-severe levels of depression. Among participants, 539 subjects (81.1\%) reported normal levels of anxiety and 125 (18.9\%) showed moderate-to-severe levels of depression. Finally, 576 participants (86.6\%) showed normal levels of stress while 88 participants (13.4\%) reported moderate-to-severe levels of stress. Descriptive statistics of the sample are reported in Table 1.

Pearson's correlations explored the relations between all the variables of interest. In general, we found significant associations between Negative affect (DASS-21), Emotion dysregulation (DERS), PIU (CIUS-7), and EDS (ESS). More specifically, all the subscales of the DASS-21 showed positive and significant correlations to each other and all the subscales of the DERS, with the only exception of the Awareness subscale, which showed no significant relations with the majority of other variables of the study. Similarly, all the subscales of the DASS-21 were significantly associated with most of the items of CIUS-7 and ESS with the only exception of the correlation between the Stress, Depression, and Anxiety subscales of the DASS-21 and items 2 and 8 of CIUS-7, and the correlation between the Stress subscale of the DASS-21 and item 3 of the CIUS-7.

The correlations between all the subscales of the DERS and the items of CIUS-7 were significant and positive, except for the Awareness subscale of the DERS which was related only to items 1 and 12 of the CIUS-7. The subscales of the 
Table I Descriptive Statistics of the Sample and Main Measures Related to Negative Affect (DASS-2I), Emotion Dysregulation (DERS), Problematic Internet Use (CIUS-7) and Daytime Sleepiness (EES)

\begin{tabular}{|c|c|c|c|c|c|c|c|c|}
\hline Variables & $\mathbf{N}$ & F (\%) & Min & Max & Mean & SD & Skewness & Kurtosis \\
\hline \multicolumn{9}{|l|}{ Gender } \\
\hline Males & 197 & 197 (29.7) & & & & & & \\
\hline Females & 467 & $467(70.7)$ & & & & & & \\
\hline Age & & & 18 & 70 & 32.13 & 11.710 & 0.625 & -1.043 \\
\hline \multicolumn{9}{|l|}{ DASS-2 I } \\
\hline Stress & & & 0 & 21 & 7.70 & 5.549 & 0.589 & -0.466 \\
\hline Anxiety & & & 0 & 21 & 3.72 & 4.370 & 1.416 & 1.644 \\
\hline Depression & & & 0 & 21 & 5.75 & 5.214 & 0.961 & 0.158 \\
\hline DASS-2I total score & & & 0 & 63 & 17.17 & $13.78 \mid$ & 0.891 & 0.142 \\
\hline \multicolumn{9}{|l|}{ DERS } \\
\hline Non-Acceptance & & & 6 & 30 & 13.36 & 5.912 & 0.849 & -0.018 \\
\hline Goals & & & 4 & 24 & 13.07 & 4.957 & 0.290 & -0.669 \\
\hline Impulse & & & 5 & 29 & 11.78 & 5.236 & 0.904 & 0.392 \\
\hline Awareness & & & 0 & 24 & 9.51 & 5.197 & 0.293 & -0.465 \\
\hline Strategies & & & 7 & 39 & 17.12 & 7.070 & 0.929 & 0.392 \\
\hline Clarity & & & 3 & 23 & 9.88 & 4.391 & 0.603 & 0.181 \\
\hline DERS total score & & & 30 & 153 & 74.72 & 23.509 & 0.654 & 0.286 \\
\hline \multicolumn{9}{|l|}{ CIUS-7 } \\
\hline ClUS I & & & 0 & 4 & 1.36 & 1.139 & 0.509 & -0.543 \\
\hline CIUS 3 & & & 0 & 4 & 0.79 & 1.047 & 1.302 & 0.943 \\
\hline CIUS 5 & & & 0 & 4 & 0.88 & 1.110 & 1.138 & 0.349 \\
\hline CIUS 7 & & & 0 & 4 & 0.48 & 0.879 & 2.038 & 3.806 \\
\hline CIUS 9 & & & 0 & 4 & 0.65 & 0.912 & 1.463 & 1.814 \\
\hline CIUS II & & & 0 & 4 & 0.50 & 0.874 & 1.956 & 3.608 \\
\hline CIUS 12 & & & 0 & 4 & 1.24 & 1.178 & 0.711 & -0.344 \\
\hline CIUS total score & & & 0 & 27 & 5.89 & 5.304 & 1.257 & 1.523 \\
\hline \multicolumn{9}{|l|}{ ESS } \\
\hline ESS I & & & 0 & 3 & 0.72 & 0.862 & 1.019 & 0.223 \\
\hline ESS 2 & & & 0 & 3 & 1.55 & 0.944 & 0.004 & -0.906 \\
\hline ESS 3 & & & 0 & 3 & 0.38 & 0.683 & 1.965 & 3.795 \\
\hline ESS 4 & & & 0 & 3 & 0.87 & 0.930 & 0.834 & -0.242 \\
\hline ESS 5 & & & 0 & 3 & 1.73 & $0.94 I$ & -0.10 & -0.982 \\
\hline ESS 6 & & & 0 & 3 & 0.10 & 0.394 & 4.436 & 21.55 \\
\hline ESS 7 & & & 0 & 3 & 0.75 & 0.873 & 0.965 & 0.067 \\
\hline ESS 8 & & & 0 & 3 & 0.12 & 0.436 & 4.025 & $|7.4|$ \\
\hline EES total score & & & 0 & 21 & 6.22 & 3.506 & 0.795 & 0.937 \\
\hline
\end{tabular}

Abbreviations: SD, standard deviation; DASS-2I, Depression Anxiety and Stress Scale; DERS, Difficulties in Emotion Regulation Scale; CIUS-7, Compulsive Internet Use Scale-7; ESS, Epworth Sleepiness Scale.

DERS were also significantly related to the items of ESS, except for the subscale Awareness of the DERS which was related only to items 4 and 5 .

The items of CIUS-7 were all significantly related to the items of ESS, with the only exception for item 2 of the ESS which was unrelated to other items of CIUS-7.

Pearson's r correlations between all the variables of interest are reported in Table 2.

\section{The Structural Equation Model}

We used a structural equation model to assess our hypothesis. The hypothesized model indicates that the positive relationships between negative affect (DASS-21) and EDS (ESS) would be sequentially and positively mediated by emotion dysregulation (DERS) and PIU (CIUS-7). The structural model is presented in Figure 2; the results of the 
Table 2 Correlations Between Negative Affect (DASS-2I), Emotion Dysregulation (DERS), Problematic Internet Use (CIUS-7) and Daytime Sleepiness (EES)

\begin{tabular}{|l|l|l|l|}
\hline & DASS-2 I & DERS & CIUS-7 \\
\hline DASS-2I & - & & \\
DERS & $0.647^{* *}$ & - & \\
CIUS-7 & $0.453^{* *}$ & $0.529^{* *}$ & - \\
EES & $0.134^{*}$ & $0.188^{* *}$ & $0.283^{* *}$ \\
\hline
\end{tabular}

Note: ${ }^{*} \mathrm{p}<0.01 ;{ }^{*} \mathrm{p}<0.001$.

Abbreviations: DASS-2I, Depression Anxiety and Stress Scale; DERS, Difficulties in Emotion Regulation Scale; CIUS-7, Compulsive Internet Use Scale-7; ESS, Epworth Sleepiness Scale.

measurement model are presented in Table 3. Latent variables are depicted in circles, while rectangles represent measured variables.

To ensure model testing, items and scales that explained less than $10 \%$ of the variance of the latent variable were excluded. $^{59}$

Negative affect is a latent variable with three indicators represented by the three subscales of DASS21, namely Depression, Anxiety, and Stress. Similarly, emotion dysregulation is a latent variable, with five indicators that correspond to the five subscales of the DERS, namely, Non-Acceptance, Goals, Impulse, Strategies, Clarity.

According to our analyses, the subscale Awareness showed a low factor loading $(-0.012)$, so it was excluded from the final model. However, this result was in line with other evidence. ${ }^{60}$ EDS was a latent variable with 6 indicators: items 2 and 8 were excluded since the items showed low factor loadings. PIU was a latent variable with seven indicators that correspond to the items of the CIUS-7.

The model showed an acceptable goodness of fit with observed data, MLR $\chi 2=284.23, \mathrm{p}<0.001 ;$ RMSEA $=0.034$; $90 \%$ C.I $[0.028-0.036]$, CFI $=0.85$; SRMR $=0.03$.

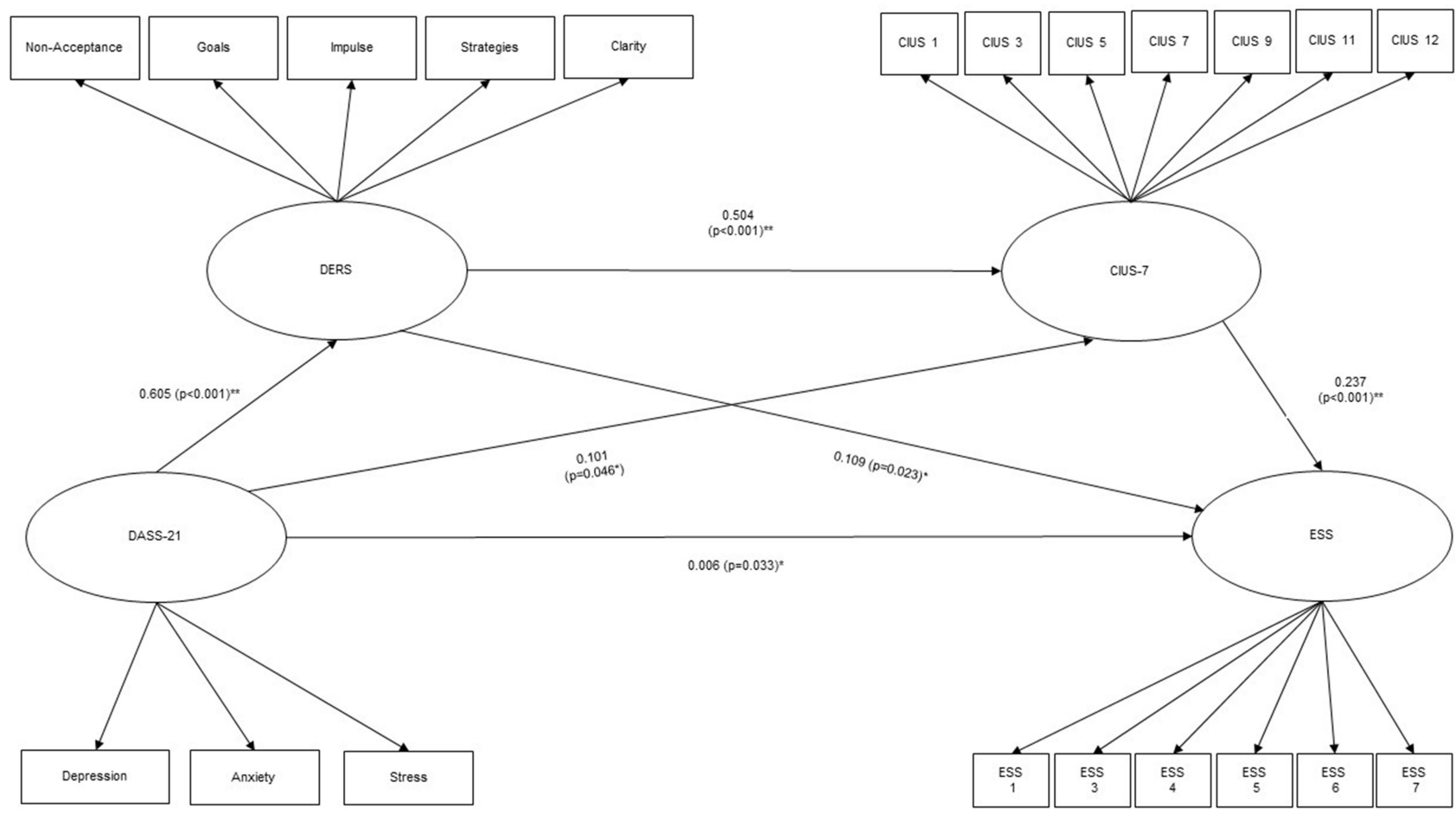

Figure 2 Standardized estimates of the mediation model.

Note: ${ }^{*}<<0.05 ; * * p<0.001$.

Abbreviations: DASS, Depression Anxiety and Stress Scale; DERS, Difficulties in Emotion Regulation Strategies; CIUS-7, Compulsive Internet Use Scale; EES, Epworth Sleepiness Scale. 
Table 3 Parameters of the Final Measurement Model

\begin{tabular}{|c|c|c|c|c|c|}
\hline Construct & Observed Variables & Latent Variable Loadings & Cronbach's Alpha & Rho_A & Adjusted $\mathbf{R}^{2}$ \\
\hline \multirow[t]{3}{*}{ DASS-2I } & Stress & 0.802 & & & \\
\hline & Anxiety & 0.833 & 0.919 & 0.922 & \\
\hline & Depression & 0.831 & & & \\
\hline \multirow[t]{5}{*}{ DERS } & Non-accept & 0.803 & & & \\
\hline & Goals & 0.822 & 0.879 & 0.885 & 0.366 \\
\hline & Impulse & 0.853 & & & \\
\hline & Strategies & 0.917 & & & \\
\hline & Clarity & 0.707 & & & \\
\hline \multirow[t]{7}{*}{ CIUS-7 } & CIUS I & 0.758 & & & \\
\hline & CIUS 3 & 0.603 & 0.919 & 0.939 & 0.330 \\
\hline & CIUS 5 & 0.686 & & & \\
\hline & CIUS 7 & 0.766 & & & \\
\hline & CIUS 9 & 0.775 & & & \\
\hline & CIUS II & 0.757 & & & \\
\hline & CIUS 12 & 0.747 & & & \\
\hline \multirow[t]{6}{*}{ ESS } & ESS I & 0.667 & & & \\
\hline & ESS 3 & 0.556 & 0.708 & 0.725 & 0.191 \\
\hline & ESS 4 & 0.587 & & & \\
\hline & ESS 5 & 0.482 & & & \\
\hline & ESS 6 & 0.629 & & & \\
\hline & ESS 7 & 0.733 & & & \\
\hline
\end{tabular}

Abbreviations: DASS-2I, Depression Anxiety and Stress Scale; DERS, Difficulties in Emotion Regulation Scale; CIUS-7, Compulsive Internet Use Scale-7; ESS, Epworth Sleepiness Scale.

The analysis of the modification indices identified that errors of the subscale Anxiety, Depression, and Stress of DASS and of items 1, 4, 6, and 7 of the ESS were correlated.

Since these variables examined highly related dimensions of the investigated constructs, their errors were allowed to covariate in the model $(r=0.28, p<0.001$ and $r=0.90, p<0.001$, for DASS subscales and ESS items, respectively). The full mediation model, including both the measurement and structural components, reported adequate indices of fit: MLR $\chi^{2}=536.50, \mathrm{p}<0.001, \mathrm{RMSEA}=0.040,90 \%$ C.I. $[0.035-0.049], \mathrm{CFI}=0.93, \mathrm{SRMR}=0.03$.

With regards to direct effects, the results indicated that higher levels of negative affect were directly associated with higher levels of emotion dysregulation and higher levels of PIU. Finally, higher levels of negative affect, higher levels of emotion dysregulation, and higher levels of PIU, were all associated with higher levels of EDS $(\beta=0.061 ; p<0.001)$.

As for the indirect effects, the mediation path from negative affect to EDS through emotion dysregulation was significant, as well as the path including only PIU as a mediator. The full sequential mediation path, including both the emotion dysregulation and PIU as mediators, showed a small but significant indirect effect $(\beta=0.072 ; p<0.05)$.

\section{Discussion}

EDS has been the focus of attention in recent years and has spurred research to identify its predictors and contributing factors. This study was conceived to explore the antecedents of EDS by assessing the relationship between negative affect, emotion dysregulation, PIU, and EDS in a sample of Italian adults from the general population. We hypothesized a significant mediating role of two-sequential mediators, emotional regulation and PIU, in the relationship between negative affect and EDS.

The results confirmed our hypothesis, showing significant associations in the expected direction between all the variables of interest. Specifically, we found a positive and significant association between negative affect and EDS. In 
addition, we also found that this relationship was partially mediated by emotion dysregulation and Internet use sequentially.

Extensive evidence are in line with our findings about the association between negative affect and EDS. ${ }^{61-64}$ Specifically, Theorell-Haglöw et al found that depression and anxiety were significant predictors of EDS. ${ }^{64}$ In line, also Smith et al, found that anxiety was a significant predictor of EDS. ${ }^{65}$

Consistently with our hypothesized model, we found a positive and significant association between negative affect and emotion dysregulation, and between emotion dysregulation and EDS. These findings were in line with previous studies showing that negative affect and emotion dysregulation were related. ${ }^{22}$ Also, research has documented a strong relationship between emotions and sleep and has suggested that emotion regulation plays an important role in the interplay between negative mood or stress and sleep problems. ${ }^{66}$

Our results showed a significant and positive association between negative affect and PIU, as well as between PIU and EDS. The positive association between negative affect and PIU is already reported in literature. ${ }^{67}$ Indeed, previous evidence suggested that individuals with PIU were more likely to show negative affect ${ }^{68}$ and sleep disturbances. $^{69}$

Similarly, as for the relation between PIU and EDS, we found additional evidence concerning the negative impact of maladaptive patterns of Internet use on sleep which was already described in literature ${ }^{70,71}$ In particular it has been previously reported that PIU is related to many sleep problems, including reduction in sleep duration, ${ }^{44-46}$ sleep onset difficulties, ${ }^{42,47}$ insomnia and EDS. ${ }^{72}$

Our findings can be interpreted in light of the compensatory model of PIU. ${ }^{34}$ There is a large body of literature suggesting that PIU can be interpreted as an "escape behavior" $60,73,74$ used to cope with distress and negative emotions in the absence of effective emotion regulation strategies. ${ }^{36}$ Individuals with difficulties in emotion regulation and who experience negative emotional states might be more likely to increase regulatory behaviors, which in turn might result dysfunctional. This implies that PIU might be a dysfunctional emotional coping. ${ }^{60,75,76}$

The current study presents important strengths, such as the consideration of a population on which the literature concerning EDS is scarce. Most previous evidence was collected in samples of young populations or clinical samples. On the contrary, in our study, we involved a non-clinical sample of Italian adult individuals from the general population. Nonetheless, the study comes with some limitations. Due to the cross-sectional design, causality could not be tested. Longitudinal studies should be done to overcome such a limitation. In addition, we involved a convenience sample of adults from the general population, and we used only self-report measures. Consequently, the results could not be extended to different samples and could be affected by biases. Moreover, in our study, we did not assess the level of exposure (for example, in terms of time spent engaging in Internet-based activities) to blue lightemitting devices, such as laptops, smartphones, and tablets. Evidence suggests that the use of these devices at bedtime has a negative effect on sleep ${ }^{77-79}$ and also impacts on melatonin secretion. ${ }^{44}$ Thus, chronic and inappropriate-timed exposure to devices can disrupt the circadian clock $^{80-82}$ eventually leading to sleep problems, which are common risk factors for EDS. ${ }^{83,84}$ Therefore, future research might consider also the role of blue-light emitting devices and their impact on sleep.

It is worth noting that the data collection for the present study has been carried out during the period of the second wave of COVID19 in Italy, which have had a strong negative impact on physical and psychological health of the Italian population, such as compromising psychological well-being, increasing depression, anxiety, stress, and reducing sleep quality, as we reported in our previous study. ${ }^{22}$ Thus, our results should be considered within the Covid-19 pandemic context, placing a limit on generalizability. Despite this, our results are still in line with the pre-Covid 19 literature.

Further replications and extensions of the study should be carried out in the future. Finally, replications in other clinical and non-clinical samples may contribute to the body of knowledge in the field.

\section{Conclusions}

According to our findings, there is a positive and significant association between negative affect and EDS. Also, we found that this relationship was partially and sequentially mediated by emotion dysregulation and PIU. 
Our study might represent an important step toward the implementation of psychological intervention for reducing EDS. Since emotion dysregulation appears to play a significant role in explaining the relationship between negative affect, PIU, and EDS, the current evidence can be used to inform psychological interventions aimed to target emotion dysregulation for treating EDS. For example, future research may evaluate whether PIU and, in turn, EDS might be prevented by developing intervention aimed at improving emotion regulation skills.

\section{Abbreviations}

EDS, excessive daytime sleepiness; OSAS, obstructive sleep apnea syndrome; PLMD, periodic limb movement disorder; PIU, problematic Internet use; ADHD, attention deficit hyperactivity disorder; DASS, depression, anxiety, and stress scale; DERS, difficulties in emotion regulation scale; ESS, epworth sleepiness scale; CIUS, compulsive Internet use scale.

\section{Ethics Statement}

All participants provided written informed consent and were informed about the purpose of the study. Data were collected and used complying with the Italian ethical standards and with the Declaration of Helsinki. The study was approved by the local ethics committee (CERIP - Comitato Etico del Centro di Ricerca e di Intervento Psicologico University of Messina, prot. n. 12106) as the University of Parma's Research Ethics Board was not established yet at the time of project evaluation.

\section{Disclosure}

GP reports participating in advisory boards for and receiving personal fees from UCB Pharma, Jazz Pharmaceuticals, Bioproject, Takeda, and Idorsia, and reports no other potential conflicts of interest in this work. The remaining authors report no conflicts of interest in this work and declare that the research was conducted in the absence of any commercial or financial relationships that could be construed as a potential conflict of interest.

\section{References}

1. Pagel JF. Excessive daytime sleepiness. Am Fam Physician. 2009;79(5):391-396.

2. American Sleep Disorders Association. The International Classification of Sleep Disorders. Rochester, MN. American Sleep Disorders Association; 1990

3. American Academy of Sleep Medicine. International Classification of Sleep Disorders. 3rd ed. Darien, IL: American Academy of Sleep Medicine; 2014.

4. Bjorvatn B, Pallesen S, Janne G, Sivertsen B, Lehmann S. Prevalence and correlates of insomnia and excessive sleepiness in adults with obstructive sleep apnea symptoms. Percept Mot Skills. 2014;118(2):571-586. doi:10.2466/15.06.PMS.118k20w3

5. Lopes C, Esteves AM, Bittencourt LRA, Tufik S, Mello MT. Relationship between the quality of life and the severity of obstructive sleep apnea syndrome. Braz J Med Biol Res. 2008;41(10):908-913. doi:10.1590/S0100-879X2008005000036

6. Ishman SL, Cavey RM, Mettel TL, Gourin CG. Depression, sleepiness, and disease severity in patients with obstructive sleep apnea. Laryngoscope. 2010;120(11):2331-2335.

7. Reishtein JL, Pack AI, Maislin G, et al. Sleepiness and relationships in obstructive sleep apnea. Issues Ment Health Nurs. 2006;27(3):319-330.

8. Mulgrew AT, Ryan CF, Fleetham JA, et al. The impact of obstructive sleep apnea and daytime sleepiness on work limitation. Sleep Med. 2007;9 (1):42-53.

9. Roth T, Roehrs TA. Etiologies and sequelae of excessive daytime sleepiness. Clin Ther. 1996;18(4):562-576.

10. Ohayon MM. How sleep and mental disorders are related to complaints of daytime sleepiness. Arch Intern Med. 1997;157(22):2645. doi:10.1001/ archinte.1997.00440430127015

11. Alcántara C, Biggs ML, Davidson KW, et al. Sleep disturbances and depression in the multi-ethnic study of atherosclerosis. Sleep. 2016;39 (4):915-925. doi:10.5665/sleep.5654

12. Adams RJ, Appleton SL, Vakulin A, et al. Association of daytime sleepiness with obstructive sleep apnoea and comorbidities varies by sleepiness definition in a population cohort of men. Respirology. 2016;21(7):1314-1321. doi:10.1111/resp.12829

13. Wilsmore BR, Grunstein RR, Fransen M, Woodward M, Norton R, Ameratunga S. Sleep habits, insomnia, and daytime sleepiness in a large and healthy community-based sample of New Zealanders. J Clin Sleep Med. 2013;9(6):559-566. doi:10.5664/jcsm.2750

14. Renko AK, Hiltunen L, Laakso M, Rajala U, Keinänen-Kiukaanniemi S. The relationship of glucose tolerance to sleep disorders and daytime sleepiness. Diabetes Res Clin Pract. 2005;67(1):84-91. doi:10.1016/j.diabres.2004.06.003

15. Lin CY, Imani V, Griffiths MD, et al. Temporal associations between morningness/eveningness, problematic social media use, psychological distress and daytime sleepiness: mediated roles of sleep quality and insomnia among young adults. J Sleep Res. 2021;30(1):1-10. doi:10.1111/ jsr.13076

16. Sameer HM, Imran N, Tarar TNKI. Association of excessive daytime sleepiness with psychological distress in medical students. Prim Care Companion CNS Disord. 2020;20(22):1. 
17. Theorell-Haglöw J, Åkerstedt T, Schwarz J, Lindberg E. Predictors for development of excessive daytime sleepiness in women: a population-based 10-year follow-up. Sleep. 2015;38(12):1995-2003. doi:10.5665/sleep.5258

18. Musetti A, Franceschini C, Pingani L, et al. Maladaptive daydreaming in an adult Italian population during the COVID-19 lockdown. Front Psychol. 2021;12:1-10. doi:10.3389/fpsyg.2021.631979

19. Gratz KL, Roemer L. Multidimensional assessment of emotion regulation and dysregulation: development, factor structure, and initial validation of the difficulties in emotion regulation scale. J Psychopathol Behav Assess. 2004;26(1):41-54. doi:10.1023/B:JOBA.0000007455.08539.94

20. Bonanno GA. Loss, trauma, and human resilience: Have we underestimated the human capacity to thrive after extremely aversive events? $A m$ Psychol. 2004;59(1):20-28. doi:10.1037/0003-066X.59.1.20

21. Blackledge JT, Hayes SC. Emotion regulation in acceptance and commitment therapy. J Clin Psychol. 2001;57(2):243-255. doi:10.1002/10974679(200102)57:2<243::AID-JCLP9>3.0.CO;2-X

22. Guerrini Usubini A, Cattivelli R, Varallo G, et al. The relationship between psychological distress during the second wave lockdown of COVID-19 and emotional eating in Italian young adults: the mediating role of emotional dysregulation. J Pers Med. 2021;11(6):569. doi:10.3390/jpm11060569

23. Bonn-Miller MO, Vujanovic AA, Boden MT, Gross JJ. Posttraumatic stress, difficulties in emotion regulation, and coping-oriented marijuana use. Cogn Behav Ther. 2011;40(1):34-44. doi:10.1080/16506073.2010.525253

24. Williams AD, Grisham JR. Impulsivity, emotion regulation, and mindful attentional focus in compulsive buying. Cognit Ther Res. 2012;36 (5):451-457. doi:10.1007/s10608-011-9384-9

25. Weinstein A, Lejoyeux M. Internet addiction or excessive internet use. Am J Drug Alcohol Abuse. 2010;36(5):277-283. doi:10.3109/ 00952990.2010 .491880

26. Beard KW, Wolf EM. Modification in the proposed diagnostic criteria for internet addiction. CyberPsychol Behav. 2001;4(3):377-383. doi:10.1089/ 109493101300210286

27. Shapira NA, Lessig MC, Goldsmith TD, et al. Problematic internet use: proposed classification and diagnostic criteria. Depress Anxiety. 2003;17 (4):207-216. doi:10.1002/da.10094

28. Schimmenti A, Musetti A, Costanzo A, et al. The unfabulous four: maladaptive personality functioning, insecure attachment, dissociative experiences, and problematic internet use among young adults. Int J Ment Health Addict. 2021;19(2):447-461. doi:10.1007/s11469-019-00079-0

29. Schimmenti A, Passanisi A, Caretti V, et al. Traumatic experiences, alexithymia, and Internet addiction symptoms among late adolescents: a moderated mediation analysis. Addict Behav. 2017;64:314-320. doi:10.1016/j.addbeh.2015.11.002

30. Hui C, Ying S, YuHui W, JiaHu H, FangBiao T. Problematic internet use in Chinese adolescents and its relation to psychosomatic symptoms and life satisfaction. BMC Public Health. 2011;11(1):1-8.

31. D'Agostino A, Covanti S, Monti MR, Starcevic V. Reconsidering emotion dysregulation. Psychiatr Q. 2017;88(4):807-825. doi:10.1007/s11126017-9499-6

32. Dimaggio G, Popolo R, Montano A, et al. Emotion dysregulation, symptoms, and interpersonal problems as independent predictors of a broad range of personality disorders in an outpatient sample. Psychol Psychother Theory Res Pract. 2017;90(4):586-599. doi:10.1111/papt.12126

33. Gioia F, Rega V, Boursier V. Problematic internet use and emotional dysregulation among young people: a literature review. Clin Neuropsychiatry. 2021;18(1):41-54. doi:10.36131/cnfioritieditore20210104

34. Kardefelt-Winther D. A conceptual and methodological critique of internet addiction research: towards a model of compensatory internet use. Comput Human Behav. 2014;31(1):351-354. doi:10.1016/j.chb.2013.10.059

35. Musetti A, Mancini T, Corsano P, Santoro G, Cavallini MC, Schimmenti A. Maladaptive personality functioning and psychopathological symptoms in problematic video game players: a person-centered approach. Front Psychol. 2019;10:1-14. doi:10.3389/fpsyg.2019.02559

36. Rogier G, Velotti P. Conceptualizing gambling disorder with the process model of emotion regulation. J Behav Addict. 2018;7(2):239-251. doi:10.1556/2006.7.2018.52

37. Tokunaga RS. Perspectives on internet addiction, problematic internet use, and deficient self-regulation: contributions of communication research. Ann Int Commun Assoc. 2015;39(1):131-161.

38. Schimmenti A, Caretti V. Psychic retreats or psychic pits?: Unbearable States of mind and technological addiction. Psychoanal Psychol. 2010;27 (2):115-132. doi:10.1037/a0019414

39. Fung TW. Identity, emotional regulation and interpersonal relationship between highly and midly internet-addictive groups. Formosa J Ment Heal. 2002;15:65-76.

40. Musetti A, Cattivelli R, Giacobbi M, et al. Challenges in internet addiction disorder: is a diagnosis feasible or not? Front Psychol. 2016;7:(JUN):18. doi:10.3389/fpsyg.2016.00842

41. Musetti A, Cattivelli R, Zuglian P, et al. Internet addiction disorder o Internet related psychopathology?. G Ital Psicol. 2017;44(2):359-382.

42. Kokka I, Mourikis I, Nicolaides NC, et al. Exploring the effects of problematic internet use on adolescent sleep: a systematic review. Int $J$ Environ Res Public Health. 2021;18(2):1-14. doi:10.3390/ijerph18020760

43. Demir G, Arslan S, Kocoglu-Tanyer D. Daytime sleepiness in university students and internet addiction as the determinant. J Addict Nurs. 2020;31 (3):153-160. doi:10.1097/JAN.0000000000000346

44. Cain N, Gradisar M. Electronic media use and sleep in school-aged children and adolescents: a review. Sleep Med. 2010;11(8):735-742. doi:10.1016/j.sleep.2010.02.006

45. Shochat T, Flint-Bretler O, Tzischinsky O. Sleep patterns, electronic media exposure and daytime sleep-related behaviours among Israeli adolescents. Acta Paediatr Int J Paediatr. 2010;99(9):1396-1400. doi:10.1111/j.1651-2227.2010.01821.x

46. Arora T, Broglia E, Thomas GN, Taheri S. Associations between specific technologies and adolescent sleep quantity, sleep quality, and parasomnias. Sleep Med. 2014;15(2):240-247. doi:10.1016/j.sleep.2013.08.799

47. Varghese NE, Santoro E, Lugo A, Madrid-Valero JJ, Ghislandi S, Torbica AGS. The role of technology and social media use in sleep-onset difficulties among Italian adolescents: cross-sectional study. J Med Internet Res. 2021;23(1):1. doi:10.2196/20319

48. Fossum IN, Nordnes LT, Storemark SS, Bjorvatn B, Pallesen S. The association between use of electronic media in bed before going to sleep and insomnia symptoms, daytime sleepiness, morningness, and chronotype. Behav Sleep Med. 2014;12(5):343-357. doi:10.1080/ 15402002.2013.819468

49. Choi K, Son H, Park M, et al. Internet overuse and excessive daytime sleepiness in adolescents: regular article. Psychiatry Clin Neurosci. 2009;63 (4):455-462. doi:10.1111/j.1440-1819.2009.01925.x 
50. Alshareef S. The impact of bedtime technology use on sleep quality and excessive daytime sleepiness in adults. Sleep Sci. 2021. doi:10.5935/19840063.20200128

51. Lovibond S, Lovibond P. Manual for the Depression Anxiety Stress Scales. Psychology Foundation of Australia; 1996.

52. Bottesi G, Ghisi M, Altoè G, Conforti E, Melli G, Sica C. The Italian version of the depression anxiety stress scales-21: factor structure and psychometric properties on community and clinical samples. Compr Psychiatry. 2015;60:170-181. doi:10.1016/j.comppsych.2015.04.005

53. Giromini L, Velotti P, De Campora G, Bonalume L, Cesare Zavattini G. Cultural adaptation of the difficulties in emotion regulation scale: reliability and validity of an Italian version. J Clin Psychol. 2012;68(9):989-1007. doi:10.1002/jclp.21876

54. Johns MW. A new method for measuring daytime sleepiness: the Epworth sleepiness scale. Sleep. 1991;14(6):540-545. doi:10.1093/sleep/14.6.540

55. Vignatelli L, Plazzi G, Barbato A, et al. Italian version of the Epworth sleepiness scale: external validity. Neurol Sci. 2003;23(6):295-300. doi:10.1007/s100720300004

56. Meerkerk GJ, Van Den Eijnden RJJM, Vermulst AA, Garretsen HFL. The Compulsive Internet Use Scale (CIUS): some psychometric properties. Cyberpsychol Behav. 2009;12(1):1-6. doi:10.1089/cpb.2008.0181

57. Lopez-Fernandez O, Griffiths MD, Kuss DJ, et al. Cross-cultural validation of the compulsive internet use scale in four forms and eight languages. Cyberpsychol Behav Soc Netw. 2019;22(7):451-464. doi:10.1089/cyber.2018.0731

58. Hu LT, Bentler PM. Cutoff criteria for fit indexes in covariance structure analysis: conventional criteria versus new alternatives. Struct Equ Model. 1999;6(1):1-55. doi:10.1080/10705519909540118

59. Kline RB. Principles and Practice of Structural Equation Modelling. 3th ed. London: The Guilford Press; 2015.

60. Blasi MDI, Giardina A, Giordano C, et al. Problematic video game use as an emotional coping strategy: evidence from a sample of MMORPG gamers. J Behav Addict. 2019;8(1):25-34. doi:10.1556/2006.8.2019.02

61. Bixler EO, Vgontzas AN, Lin HM, Calhoun SL, Vela-Bueno A, Kales A. Excessive daytime sleepiness in a general population sample: the role of sleep apnea, age, obesity, diabetes, and depression. J Clin Endocrinol Metab. 2005;90(8):4510-4515. doi:10.1210/jc.2005-0035

62. Chellappa SL, Araújo JF. Excessive daytime sleepiness in patients with depressive disorder. Rev Bras Psiquiatr. 2006;28(2):126-129. doi:10.1590/ S1516-44462006000200010

63. Mume CO. Excessive daytime sleepiness among depressed patients. Libyan J Med. 2010;5(1):1-4.

64. Theorell-Haglöw J, Lindberg E, Janson C. What are the important risk factors for daytime sleepiness and fatigue in women?. Sleep. 2006;29 (6):751-757.

65. Smith S, Rossdale J, Serry Y, Sekaran A, Drakatos P, Steier J. Multiple dimensions of excessive daytime sleepiness. J Thorac Dis. 2018;10(5): S170-6.

66. Vandekerckhove M, Wang YL. Emotion, emotion regulation and sleep: an intimate relationship. AIMS Neurosci. 2018;5(1):1-17.

67. Musetti A, Terrone G, Schimmenti A. An exploratory study on problematic internet use predictors: Which role for attachment and dissociation?. Clin Neuropsychiatry. 2018;15(1):35-41.

68. Shaw M, Black DW. Internet addiction: definition, assessment, epidemiology and clinical management. CNS Drugs. $2008 ; 22(5): 353-365$.

69. Singh LK, Suchandra KH, Pattajoshi A, et al. Internet addiction and daytime sleepiness among professionals in India: a web-based survey. Indian J Psychiatry. 2019;61(3):265-269.

70. Guo L, Luo M, Wang WX, et al. Association between problematic Internet use, sleep disturbance, and suicidal behavior in Chinese adolescents. J Behav Addict. 2018;7(4):965-975.

71. Do KY, Lee KS. Relationship between problematic internet use, sleep problems, and oral health in Korean adolescents: a national survey. Int J Environ Res Public Health. 2018;15(9):1-14.

72. Park MH, Park S, Jung KI, Kim JI, Cho SC, Kim BN. Moderating effects of depressive symptoms on the relationship between problematic use of the Internet and sleep problems in Korean adolescents. BMC Psychiatry. 2018;18(1):1-8.

73. Deleuze J, Maurage P, Schimmenti A, Nuyens F, Melzer A, Billieux J. Escaping reality through videogames is linked to an implicit preference for virtual over real-life stimuli. J Affect Disord. 2019;245:1024-1031.

74. Pettorruso M, Valle S, Cavic E, Martinotti G, Di Giannantonio M, Grant JE. Problematic Internet use (PIU), personality profiles and emotion dysregulation in a cohort of young adults: trajectories from risky behaviors to addiction. Psychiatry Res. 2020;289:113036.

75. Giardina A, Di Blasi M, Schimmenti A, et al. Online gaming and prolonged self-isolation: evidence from Italian gamers during the COVID-19 outbreak. Clin Neuropsychiatry. 2021;18(1):65-74.

76. Giardina A, Starcevic V, King DL, Schimmenti A, Di Blasi M, Billieux J. Research directions in the study of gaming-related escapism: a commentary to Melodia, Canale, and Griffiths (2020). Int J Ment Health Addict. 2021;0123456789. doi:10.1007/s11469-021-00642-8

77. Rahman SA, St. Hilaire MA, Lockley SW. The effects of spectral tuning of evening ambient light on melatonin suppression, alertness and sleep. Physiol Behav. 2017;177:221-229. doi:10.1016/j.physbeh.2017.05.002

78. Grønli J, Byrkjedal IK, Bjorvatn B, Nødtvedt O, Hamre B, Pallesen S. Reading from an iPad or from a book in bed: the impact on human sleep. A randomized controlled crossover trial. Sleep Med. 2016;21:86-92. doi:10.1016/j.sleep.2016.02.006

79. Heo JY, Kim K, Fava M, et al. Effects of smartphone use with and without blue light at night in healthy adults: a randomized, double-blind, cross-over, placebo-controlled comparison. J Psychiatr Res. 2017;87(2017):61-70. doi:10.1016/j.jpsychires.2016.12.010

80. Touitou Y, Reinberg A, Touitou D. Association between light at night, melatonin secretion, sleep deprivation, and the internal clock: health impacts and mechanisms of circadian disruption. Life Sci. 2017;173:94-106. doi:10.1016/j.1fs.2017.02.008

81. Rångtell FH, Ekstrand E, Rapp L, et al. Two hours of evening reading on a self-luminous tablet vs. reading a physical book does not alter sleep after daytime bright light exposure. Sleep Med. 2016;23:111-118. doi:10.1016/j.sleep.2016.06.016

82. Bedrosian TA, Nelson RJ. Timing of light exposure affects mood and brain circuits. Transl Psychiatry. 2017;7(1). http://dx.doi.org/10.1038/tp.2016. 262

83. Bhat S, Pinto-Zipp G, Upadhyay H, Polos PG. "To sleep, perchance to tweet": in-bed electronic social media use and its associations with insomnia, daytime sleepiness, mood, and sleep duration in adults. Sleep Health. 2018;4(2):166-173. doi:10.1016/j.sleh.2017.12.004

84. Slater G, Steier J. Excessive daytime sleepiness in sleep disorders. J Thorac Dis. 2012;4(6):608-616. 


\section{Publish your work in this journal}

Nature and Science of Sleep is an international, peer-reviewed, open access journal covering all aspects of sleep science and sleep medicine, including the neurophysiology and functions of sleep, the genetics of sleep, sleep and society, biological rhythms, dreaming, sleep disorders and therapy, and strategies to optimize healthy sleep. The manuscript management system is completely online and includes a very quick and fair peer-review system, which is all easy to use. Visit http://www.dovepress.com/testimonials.php to read real quotes from published authors.

Submit your manuscript here: https://www.dovepress.com/nature-and-science-of-sleep-journal 